\title{
Development of SSR markers via de novo transcriptome assembly in Akebia trifoliata (Thunb.) Koidz.
}

\begin{tabular}{|c|c|}
\hline Journal: & Genome \\
\hline Manuscript ID & gen-2019-0068.R2 \\
\hline Manuscript Type: & Article \\
\hline $\begin{array}{r}\text { Date Submitted by the } \\
\text { Author: }\end{array}$ & 21-Aug-2019 \\
\hline Complete List of Authors: & $\begin{array}{l}\text { Niu, Juan; Chinese Academy of Agricultural Sciences Institute of Bast } \\
\text { Fiber Crops } \\
\text { Wang, Yujuan; Jiangxi Academy of Forestry of China } \\
\text { Shi, Yaliang; Chinese Academy of Agricultural Sciences Institute of Bast } \\
\text { Fiber Crops } \\
\text { Wang, Xiaofei; Chinese Academy of Agricultural Sciences Institute of } \\
\text { Bast Fiber Crops } \\
\text { Sun, Zhimin; Chinese Academy of Agricultural Sciences Institute of Bast } \\
\text { Fiber Crops } \\
\text { Huang, Kunyong; Chinese Academy of Agricultural Sciences Institute of } \\
\text { Bast Fiber Crops } \\
\text { Gong, Chun; Chinese Academy of Agricultural Sciences Institute of Bast } \\
\text { Fiber Crops } \\
\text { Luan, Mingbao; Chinese Academy of Agricultural Sciences Institute of } \\
\text { Bast Fiber Crops } \\
\text { Chen, Jianhua; Chinese Academy of Agricultural Sciences Institute of } \\
\text { Bast Fiber Crops }\end{array}$ \\
\hline Keyword: & SSR, Transcriptome, Akebia trifoliata, Accession identification \\
\hline $\begin{array}{r}\text { Is the invited manuscript for } \\
\text { consideration in a Special } \\
\text { Issue? : }\end{array}$ & Not applicable (regular submission) \\
\hline
\end{tabular}

\section{SCHOLARONE \\ Manuscripts}


1 Development of SSR markers via de novo transcriptome assembly in Akebia trifoliata (Thunb.) Koidz.

2 Juan Niu ${ }^{1}$, Yujuan Wang ${ }^{2}$, Yaliang Shi ${ }^{1}$, Xiaofei Wang ${ }^{1}$, Zhimin Sun ${ }^{1}$, Kunyong Huang ${ }^{1}$, Chun Gong ${ }^{2}$, Mingbao Luan ${ }^{1 *}$, Jianhua Chen ${ }^{1 *}$

5 of Agriculture, Changsha 410205, P. R. China

$6 \quad$ 2Jiangxi Academy of Forestry of China, Nanchang 330033, P. R. China

7 Juan Niu and Yujuan Wang contributed equally to this work.

8 * Corresponding authors: Jianhua Chen

9 Institute of Bast Fiber Crops, Chinese Academy of Agricultural Sciences, Xianjiahu West Road, Changsha 410205, Hunan Province, China

$10 \quad$ E-mail addresses: cjhbt@sina.com

11 * Corresponding authors: Mingbao Luan; E-mail addresses: luanmingbao@caas.cn 
12 Abstract: Owing to its high nutritive, economic, and medicinal values, Akebia trifoliata has received increased attention, making worthy of being used as a new fruit crop for further domestication and commercialization in China. However, molecular research of A. trifoliata has lagged as investigations of its genomic resources and

14 molecular markers are rare. In this study, a cDNA library of $A$. trifoliata leaves was sequenced using the Illumina NovaSeq 6000 sequencing system. 101417 transcripts, 63757 unigenes, and 9494 simple sequence repeats were assembled and identified from the transcriptome datasets. The majority of the SSRs were di- and trinucleotide repeats. Length and number of SSR motifs ranged from 15 to 66 , and 5 to $48 \mathrm{bp}$, respectively. Of which, the A/T mononucleotide motif and AG/TC and

$17 \mathrm{CT} / \mathrm{GA}$ dinucleotide motifs were the most abundant. Furthermore, $100 \mathrm{SSR}$ primers were randomly selected to validate amplification and polymorphism, and $88 \mathrm{~A}$. trifoliata accessions were definitively distinguished by 49 primers. With the Qinling mountains and Huaihe River line as the boundaries, the northern and southern accessions were clustered into different groups, but no clear geographical patterns (city or origin) were observed in the southern accessions. These newly identified molecular markers may provide a foundation for the genetic identification and diversity analysis and marker-assisted selection breeding in Akebia species. 


\section{Introduction}

Akebia trifoliata (Thunb.) Koidz $(2 \mathrm{n}=16)$ is a wild perennial woody liana, and the A. trifoliata plants are monoecious with flowers functionally unisexual and self-incompatible (Zou et al. 2018; Li et al. 2019). It belongs to the Lardizabalaceae family, that is mainly distributed throughout China and Japan, and is also commonly known in China as August melon, August bar, Bayuezha, and wild banana (Liu et al. 2007; Du et al. 2012). A. trifoliata has been used in traditional Chinese medicine (TCM) for more than 2000 years (Wang et al. 2014). Previous research has shown that its various parts, such as the roots, stems, leaves, flowers, and fruits, may be edible, medicinal, and ornamental, making it a desirable plant for consumers (Zhou et al. 2017). For example, its fruits and stems are used in TCM to promote diuresis, and as an antiphlogistic to stimulate blood circulation (Kawata et al. 2007); its dried young leaves can be used as tea leaves, and the fresh fruits, which are rich in saccharides, vitamins, and amino acids and serve as a kind of high-value and health fruit (Du et al. 2012; Wang et al. 2015), can be consumed directly or used to make juice, sugar, and vinegar (Jiang et al. 2012). Furthermore, the oil of seeds exhibits an abundance of nutritional properties and is commonly used as an edible oil in southern China (Kitaoka et al. 2009). Owing to its highly nutritive, economic, and medicinal values, A. trifoliata is currently being widely cultivated and rapidly developed as a commercial species in many regions of China (Li et al. 2010a; Li et al. 2010b). 
37 Owing to their high polymorphism, reliability, and rapid and ready detection, simple sequence repeat markers (SSR) have proven to be powerful tools in conservation biology and molecular ecological studies (Ohbayashi et al. 2019). However, until now, a mere 145 nucleotide sequences and no expressed sequence tags (EST)

39 sequences had been deposited for $A$. trifoliata in the National center for biotechnology information (NCBI) database, and only 125 unreviewed sequences have been

40 added to the UniProt database. Furthermore, only 11 microsatellite loci were reported from an AC-enriched genomic library of A. trifoliate ssp. australis, to be

41 utilized for population genetic diversity analysis of different Akebia species (Li et al. 2009; Li et al. 2019). As the 11 reported markers were developed from partial

42 genomic DNA sequences, their isolation was time-consuming, laborious, and expensive. Previous studies indicated that most of the reported genomic SSR markers have lower rate of transferability than EST-SSR markers to related species, and were difficult to correlate with the target agronomic traits (Shi et al. 2017). The low percentage of polymorphic markers identified for A. trifoliata has greatly limited research into its genetic diversity, accession preservation, and molecular breeding. Additionally, although the use of transcriptome sequencing has recently been reported in A. trifoliata (Yang et al. 2016), very few molecular markers have been developed. 
number of SSR markers from the assembled transcripts. We randomly selected 100 SSR primers to test for their utilization in the study of the genetic diversity of $A$. trifoliata accessions. These findings could provide new insights for genetic research and marker-assisted selection (MAS) breeding of Akebia plants.

\section{Materials and methods}

\section{Plant materials}

The Qinling mountains and Huaihe River line (Qinling-Huaihe line) form the geographical dividing line between the northern and southern regions of China. They run west to east, stretching across the Gansu, Shaanxi, and Henan Provinces. To the north of the Qinling-Huaihe line, the climate is temperate, rivers freeze, and the leaves seasonally turn yellow and fall from the trees. To the south of the Qinling-Huaihe line, there is a subtropical monsoon climate, the rivers do not freeze, and the trees stay green all year round (Li et al. 2015). Nanyang city in Henan Province is on the Qinling Huaihe line boundary, but it has a subtropical monsoon climate and

59 is grouped with the southern region. For this study, a total of 88 wild accessions of $A$. trifoliata were collected from 8 provinces including 16 regions of China and

60 their topographic altitudes ranged from 33 to $1646 \mathrm{~m}$ above sea level (Fig. 1). According to the divisions of the Qinling-Huaihe line, the accessions collected from the

61 cities of Luoyang and Dengfeng were categized as northern resources. While the accessions collected from Changsha, Changde, Zhangjiajie, Zhuzhou, Nanchang, 
62 Ganzhou, Dazhou, Sanming, Hanzhong, Shangrao, Ningguo, Nanyang city, Enshi, and the Xiangxi autonomous prefecture were categized as southern resources. All the accessions collected $(2 \mathrm{n}=16)$ of $A$. trifoliata were wild, monoecious with flowers functionally unisexual and self-incompatible. The accessions from 1-36 were

64 cultivated in preservation sites, including Hunan academy of agricultural sciences (HAAS) and Jiangxi academy of forestry (JXAF), and the accessions 37-88 were planted in the resources nursery of Institute of bast fiber crops (IBFC). However, owing to a lack of systematic planting maps and uncertain recordings, the accessions collected from HAAS and JXAF (accounting for $37.5 \%$ of all accessions) was not systematically recorded. The geographical origin and biological

67 characteristics of the accessions in these regions was also not available. In addition, for the purple peel and higher fruit quality of the accession, the fresh young leaves from 'Dazhou No.3' were used to construct a transcriptome library. Furthermore, 88 accessions were used to examine the polymorphisms of the detected SSR markers in our study (Table 1). All the fresh young leaves were collected and immediately frozen in liquid nitrogen and stored at $-80{ }^{\circ} \mathrm{C}$, until further analysis.

\section{RNA and DNA extraction}

71 The total RNA was isolated from 'Dazhou No.3' using the cetyltrimethylammonium bromide (CTAB) methods, as described by Niu et al. (2018). Total genomic

72 DNA was extracted from each of the 88 leaf samples using the CTAB method with minor modifications, according to Xu et al. (2013). The quality and the 73 concentration of the total RNA and DNA was evaluated by electrophoresis using a $1.0 \%$ agarose gel and a NanoDrop 2000C Spectrophotometer (Thermo Fisher

74 Scientific, Waltham, MA, USA). 


\section{Library construction and Illumina sequencing}

First, the total RNA of the sample 'Dazhou No.3' was collected and oligo (dT) beads were used to enrich the poly (A) mRNA. The enriched mRNA was then broken into short fragments using a fragmentation buffer. Subsequently, the first-strand cDNA was synthesized by random hexamers using short fragments as templates, and then the second-strand cDNA was synthesized by using buffer, dNTPs, RNase H, and DNA polymerase I. The synthesized double-stranded cDNA fragments were then purified by magnetic beads and eluted with EB buffer for end-repair and the addition of poly (A). The short fragments were connected with sequencing adapters, and suitable fragments were selected as templates with magnetic beads for PCR amplification and agarose gel electrophoresis. Finally, cDNA libraries were built and sequenced using the Illumina NovaSeq 6000 platform of Anhui Microanaly Genetech Company (Anhui, China).

\section{De novo assembly of the transcriptome}

To ensure the accuracy of the de novo assembly and subsequent analysis, the raw sequence data reads were processed prior to the assembly of the $A$. trifoliata transcriptome. The raw reads were initially processed using custom Perl scripts in fastq format and the filtering process was divided into 3 parts: (1) the excess "N" nucleotides, with more than $10 \%$ of the bases, and reads with low-quality bases $(\mathrm{Q}<30)$, were filtered out; (2) reads with less than 15 bases or more than $40 \%$ poor bases were removed; (3) the adaptor sequences were filtered out. Then the clean reads were obtained by filtering out these adapter and low-quality sequences from 

2011). The Trinity software used three independent modules, including Inchworm, Chrysalis, and Butterfly, and default parameter settings; transcripts shorter than

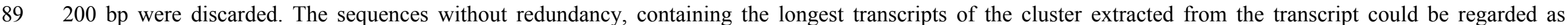

90 unigenes.

\section{$91 \quad$ Gene annotation}

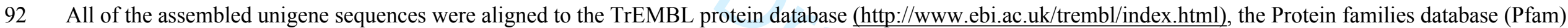

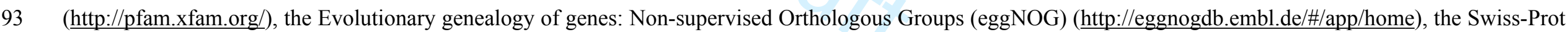

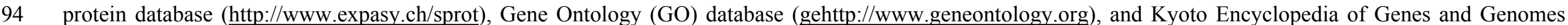

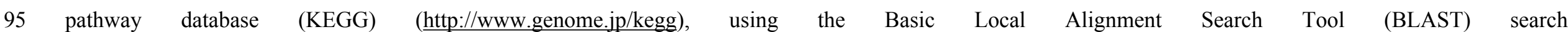

96 (https://www.arabidopsis.org/cgi-bin/Blast/TAIRblast.pl) with an $E$-value of $10^{-5}$.

97 Simple sequence repeat (SSR) identification

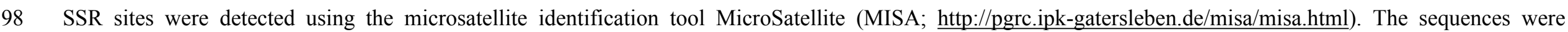


searched for perfect mononucleotide, dinucleotide, trinucleotide, tetranucleotide, pentanucleotide, and hexanucleotide motifs, with a minimum of fifteen, eight, five, four, three, and three repeats, respectively. Primer pairs were randomly selected and designed in a further study using Primer Premier 3.0 (http://sourceforge.net/projects/primer3) in the flanking regions of the SSRs. In order to acquire a high probability of amplification, the primer design parameters were set as follows: the primer length was $18-23 \mathrm{bp}$ with an optimal length of $21 \mathrm{bp}$; the PCR products were $100-300 \mathrm{bp}$, the annealing temperature was $52-58{ }^{\circ} \mathrm{C}$ with an optimal temperature of $55{ }^{\circ} \mathrm{C}$, and the GC percentage was $40-60 \%$, with an optimal GC content of $50 \%$. Primers were synthesized by Qingke Biotech

104 (Beijing, China).

\section{EST-SSR marker characterization and validation}

To analyze the SSR polymorphisms, the identified SSR markers were amplified and validated with 88 accessions of $A$. trifoliata. PCR was performed in a total reaction volume of $20 \mu \mathrm{L}$ containing $1 \mathrm{U}$ Taq DNA polymerase (TIANGEN Biotech, Beijing, China), $1 \times$ PCR Buffer, $1.5 \mathrm{mM} \mathrm{MgCl} 2,0.52 \mathrm{mM}$ dNTPs (TIANGEN Biotech, Beijing, China), $0.4 \mu \mathrm{M}$ of each primer, and $20 \mathrm{ng}$ of genomic DNA. PCR was carried out on a Bio-Rad T100 

recorded as the capital letter A, followed by B, C, D, etc., and different letters were used to record different morphological variants. If only one band type was found

114 by a pair of primers, the accession was recorded as homozygous. The major allele frequency (MAF), the number of alleles $\left(N_{a}\right)$, genetic distance, observed heterozygosity $\left(H_{o}\right)$, and the polymorphic information content (PIC) were calculated and constructed using PowerMarker v3.25 (Liu et al. 2005). The clustering tree was constructed based on Nei's genetic distance, which was calculated by PowerMarker and visualized with the MEGA version 7.0 and iTOL (https://itol.embl.de/) (Luo et al. 2018). The population structure of the accessions was determined using the model with the admixture and the allele variation frequency correlation model of the software program Structure (version 2.3.4) (Pritchard et al. 2000). The software program Structure Harvester V6.0 was used to determine the optimal $K$ value

\section{9 (Earl et al. 2012).}

\section{Availability of sequencing data} accession numbers are PRJNA525587 and SRP187511, respectively. 


\section{Results}

\section{Sequencing and de novo assembly of the $A$. trifoliata transcriptome}

A total of 50364426 raw reads $(7.555 \mathrm{~Gb})$ were produced for $A$. trifoliata using Illumina sequencing, and 25049450 clean reads were obtained after filtering and trimming of the low quality and adaptor sequences, and the ambiguous ("N") nucleotides. The clean reads totaled $7.205 \mathrm{~Gb}$ with a mean length of 143.81 bp, the GC and Q30 content was $45.10 \%$ and $92.56 \%$, respectively. A total of 101417 transcripts were assembled from the clean reads after the de novo assembly, with an N50 of $1692 \mathrm{bp}$, and an average length of $983.28 \mathrm{bp}$. The length distributions of the unigenes are shown in Fig. S1. A total of 63757 unigene sequences were generated from the transcriptome library, with an N50 of $1487 \mathrm{bp}$, and an average length of $769.89 \mathrm{bp}$ (Table 2). Moreover, based on the BLASTx algorithm with an $E$-value threshold of $10^{-5}, 19661$ (30.84 \%) unigenes were annotated in the Pfam database, 27772 (43.56 \%) in TrEMBL, 20 917 (32.81 \%) in eggNOG, 19096 (29.95 \%) in Swiss-Prot, 22798 (35.76 \%) in GO, and 27309 (42.83\%) in KEGG (Table 3); suggesting that the TrEMBL database provided the greatest number of annotations. Nelumbo nucifera $(13247,47.70 \%)$ in the TrEMBL database. 


\section{Frequency and distribution of EST- SSRs in $A$. trifoliata}

A total of 9494 SSRs were identified from 63757 unigene sequences using the MISA tool. The frequencies of these SSR repeat types were different; the dinucleotide motifs were the most common type of repeat motif, with a frequency of $42.35 \%$, followed by the trinucleotide $(30.02 \%)$, mononucleotide $(24.65 \%)$, and tetranucleotide (2.18\%) motifs (Fig. 2A, Table 4). The lengths of the SSR motifs ranged from 15 to 66 bp, with 15 to 19 bp (60.80 \%) being the most abundant SSR motif lengths, followed by SSR motifs with lengths ranging from 20 to 24 bp (27.63\%). However, SSR motifs with lengths that ranged from 25 to 29 bp, 30 to 34 bp, and those composed of greater than $35 \mathrm{bp}$, accounted for only 4.14, 4.22, and $3.21 \%$ of the total number of SSRs, respectively (Fig. 2B).

The number of repeats in the SSR motifs ranged from 5 to 48, and 10-14 was the most abundant number of repeats, accounting for $48.43 \%$ of the SSRs, followed by 5-9 repeats $(37.12 \%)$. The next most common numbers of repeats were 15-19 (11.00\%) and 20-24 (2.68 \%) (Fig. 3A). The largest number of repeats for each repeat length was $48,33,20,12,10$, and 8 for the mono, di-, tri-, tetra-, penta-, and hexanucleotides, respectively.

A total of 155 types of repeats were identified, where 2, 6, 30, 29, 34, and 33 different types of repeat motifs were detected within the mono-, di-, tri-, tetra-, penta-, and hexanucleotide repeats, respectively. In the mononucleotide repeats, A/T was the most abundant, and accounted for $24.39 \%$ of all motifs. The AG/TC repeat was dominant in the di-motifs and accounted for $19.04 \%$ of all motifs. Furthermore, each of the repeats CT/GA (15.10 \%), AC/TG (3.27 \%), AT/TA 

2340 mononucleotide motifs, $2316(98.97 \%)$ were composed of A/T repeats. Among the 4021 dinucleotide motifs, 1808 (44.96 \%) and 1434 (35.66 \%), were

\section{Validation of SSR markers in $A$. trifoliata}

The ID, length of the unigenes, SSR type, SSR loci, and the sequences of the SSR loci are shown in Table S2. To validate the amplification efficacy and polymorphisms of the putative SSR markers, 100 primers were randomly selected, synthesized, and screened based on the SSRs. Of these, 72 primers successfully produced clear bands with polymorphisms, and 28 primers produced banding patterns without polymorphisms in 4 accessions, as visualized by PAGE. Moreover, 49 of the 72 primers produced the expected bands and showed polymorphisms in all 88 of the $A$. trifoliata accessions. A total of 136 alleles were detected, with an average of 3 alleles per locus, where the number of alleles per locus ranged from 2 to 4 . $H_{0}$ ranged from 0 to 0.94 (with a mean of 0.22 ), and the PIC values varied from 0.09 to 0.63 (with a mean of 0.43 ). MAF varied from 0.36 to 0.95 , with an equilibration of 0.60 , and the gene diversity ranged from 0.09 to 0.69 , with an 
P023 are shown as examples (Fig. S2). These results indicate that the polymorphic SSRs derived from the RNA-seq behave effectively as markers and are suitable for inferring population genetic structure in A. trifoliata.

\section{Analysis of genetic diversity}

The genetic relationships of the $88 \mathrm{~A}$. trifoliata accessions were analyzed based on the allele polymorphisms of the 49 SSR primers, and the accessions were grouped into four main clades, using Powermarker software. Clade I contained 27 accessions, including LuoyangNo.1-No.11, DengfengNo.2-No.14, JF05, DazhouNo.1, and No.3. Clade II contained 12 accessions, including ZhangjiajieNo.1, HanzhongNo.1-No.5, JF06, HA1, HA10, JF02, JF04, and JF11. Clade III contained 18 accessions, including HA06-HA08, HA12-HA15, HA18, ShangraoNo.1-No.2, NingguoNo.1, JF08-JF10, and JF14-JF17. Clade IV contained 31 accessions, including HA02-HA05, HA09, HA11, HA16-HA17, HA19, JF01, JF07, ChangdeNo.1-No.4, DengfengNo.1, NanyangNo.1, DazhouNo.2, DazhouNo.4-No.6, ZhangjiajieNo.2, SanmingNo.1, EnshiNo.1-No.5, GanzhouNo.1, ZhuzhouNo.1, and XiangxiNo.1 (Fig. 4). The 88 accessions were clustered into different groups. always cluster into the same group, while those from different locations did sometimes cluster together. 
The population structure of the 88 accessions was also examined, based on the SSR markers using the Structure software program. The results of the inferred clustering, completed using a $K$-value (population number) of 2-10, revealed that the most significant change in likelihood occurred when the $K$ value increased from

2 to 4 , and that the highest Delta $K(\Delta K)$ value was observed between $K=2$ and $K=4$. Moreover, when $K=4$, the northern accessions could be completely separated

from the southern accessions. The results from the dendrogram analysis were similar, as four main groups were cultured, and all parameters suggested that the four-clade model sufficiently explained the genetic structures of the 88 accessions (Fig. 5).

\section{Discussion}

\section{Unigene assembly and annotation}

A. trifoliata is rich in nutrients, has health benefits, and other newly discovered fruit properties in the primary stages of its domestication, that make it worthy of 

expression (Hou et al. 2018), developing species molecular markers, and phylogenetic relationships (Choudhary et al. 2018; Yang et al. 2018). In the present study, a total of 25049450 clean reads were obtained using transcriptome sequencing, and the GC content and Q30 were $45.10 \%$ and $96.31 \%$, respectively. Moreover, 63 757 unigenes with an N50 of $1487 \mathrm{bp}$, and an average length of $769.89 \mathrm{bp}$ were successfully assembled from the $A$. trifoliata transcriptome (Table 2). The lengths obtained in this study were longer than those of other Ranunculales, such as Dysosma versipellis (938 bp and 409 bp, respectively) (Guo et al. 2014), Sargentodoxa cuneata (an average length of 255 bp) (Sun et al. 2016), and D. aurantiocaulis (807 bp and 531 bp, respectively) (Mao et al. 2016). These differences indicate the high-quality nature of the transcriptome sequencing and de novo assembly performed in the present study on $A$. trifoliata. results are similar to values reported for D. versipellis (27.98\%) (Guo et al. 2014) and Rhododendron (43.22\%) (Xing et al. 2017), but less than the values reported trifoliata may provide useful reference data for the molecular research of Akebia species.

\section{Markers identification and characterization of SSRs}


As one of the most widely used molecular markers in crops and fruits, the clear advantages of SSR markers (i.e., a high degree of polymorphism, good repeatability, and ease of use) play a key role in their broad application for studies on population genetic structure and diversity, evolution, genetic mapping, comparative genomics, etc. (Hu et al. 2017). Despite these advantages, few SSR markers and nucleotide sequences—and no EST sequences—-have been reported in the GenBank database for A. trifoliata, which is a major obstacle for the development of molecular marker-assisted selection in this species. Transcriptome sequences have become the preferred method for the development of molecular markers, particularly in non-model species, such as A. trifoliata. A total of 9494 SSRs were identified in the present study, where the dinucleotide motifs were the most common type of repeat motif, with a frequency of $42.35 \%$, followed by trinucleotides ( $30.02 \%$ ). By contrast, tetra-, penta-, and hexa-nucleotide repeats were far less common $(2.18,0.45$, and $0.35 \%$, respectively) (Table 4 , Fig. 2A). These results are consistent with the SSR results observed in the transcriptome of D. versipellis (Guo et al. 2014). In addition, the distributions of the types of microsatellite repeats were also similar to those of other plants, such as in D. versipellis and sweet potato, suggesting that the AG/TC, CT/GA, and AGA/TCT, AAG/TTC repeats may be the predominant motifs in dicotyledonous plants (Guo et al. 2014; Wang et al. 2010). Additionally, there was no GC/CG motif, which was consistent with the observation that hairpins structures (Eustice et al. 2008).

\section{Validation of the predicted SSR markers}


In order to validate the amplification quality and polymorphisms of the SSRs in the present study, a total of 100 randomly generated primers were validated across 88 accessions. Of these, 72 primers (72\%) successfully produced clear bands with polymorphisms, $28(28 \%)$ primers produced bands without polymorphism in 4 accessions. Forty-nine of the 72 primers generated the expected bands (49\%) with polymorphism in 88 A. trifoliata accessions. The polymorphic ratio (49 \%) of the primers was similar to the results found in species of the subfamily Podophylloideae (51.0 \%) (Mao et al. 2016) but higher than the ratios observed in D. versipellis (46.3\%) (Guo et al. 2014) and S. cuneata (36.2\%) (Sun et al. 2016). This may be explained by the presence of introns and deletions within the amplicons, assembly errors, or low specificity because of unintentional selection of these primers (Wu et al. 2013; Chen et al. 2017). Moreover, the corresponding value for the average alleles per locus was 3 of A. trifoliata, which is like the average values for EST-SSR markers reported in previous studies on D. versipellis (3.32) and D. pleiantha (3.50). The PIC values, which varied from 0.09 to 0.63 (with a mean of 0.43) (Table 4), were lower than the range observed in populations of D. versipellis (from 0.009 to 0.750 ; mean 0.496 ) reported by Guo et al. (2014). The lower level of allelic diversity at the sites of the SSRs could be the result of the random selection of

Lardizabalaceae and Ranunculales. 
The long-term over-harvesting and habitat degradation of the natural A. trifoliata populations has resulted in their dramatic decline and thus, reasonable conservation strategies and restoration of the accessions is urgently needed (Li et al. 2010a). Furthermore, the lack of systematic planting maps or reliably recorded data makes it difficult to identify the available accessions collected from HAAS and JXAF. Their geographical origins and biological characteristics were also not available. In order to improve the conservation and sustainable use of this species, it is necessary to analyze the phylogenetic relationships of these accessions. The 88 A. trifoliata accessions were found to be grouped within four main clades by the clustering analysis, which is in accord with the results of the population structure analyses (Fig. 4 and Fig. 5). According to the divisions of the Qinling-Huaihe line, the northern and southern accessions were clustered into different groups, but no clear geographical patterns (city or origin) were observed among the southern accessions. These results showed that not only the geographical environment had a certain effect on the differences of the accessions, but other factors such as altitude, climatic, differences biological characteristics, gene flow, and genetic drift may also affect the genetic diversity of the accessions (Wróblewska 2014). conservation genetics, linkage mapping, genetic breeding, and MAS breeding for this commercially and medicinally valuable species. 

Agricultural Science, Technology Innovation Program (ASTIP) of CAAS (Grant No.2017IBFC).

\section{References}

Chen, H., Wang, L., Liu, X., Hu, L., Wang, S., and Cheng, X. 2017. De novo transcriptomic analysis of cowpea (Vigna Unguiculata L. Walp.) for genic SSR marker development. BMC Genet 18(1): 65-76. doi:10.1186/s12863-017-0531-5. PMID: 28693419.

Choudhary, S., Thakur, S., Najar, R.A., Majeed, A., Singh, A., and Bhardwaj, P. 2018. Transcriptome characterization and screening of molecular markers in ecologically important Himalayan species (Rhododendron arboreum). Genome 61(6): 417-428. doi:10.1139/gen-2017-0143. PMID: 29658317.

Deng, K., Deng, R., Fan, J., and Chen, E. 2018. Transcriptome analysis and development of simple sequence repeat (SSR) markers in Zingiber striolatum Diels. Physiol Mol Biol Plants 24(1): 125-134. doi:10.1007/s12298-017-0485-0. PMID: 29398844.

Du, Y., Jiang, Y., Zhu, X., Xiong, H., Shi, S., Hu, J., et al. 2012. Physicochemical and functional properties of the protein isolate and major fractions prepared from Akebia trifoliata var. australis seed. Food Chem 133(3): 923-929. doi:10.1016/j.foodchem.2012.02.005. 
Earl, D.A. 2012. STRUCTURE HARVESTER: a website and program for visualizing STRUCTURE output and implementing the Evanno method. Conserv Genet Resour 4(2): 359-361. doi:10.1007/s12686-011-9548-7. papaya. Tree Genet Genomes 4(2): 333-341. doi:10.1007/s11295-007-0112-2. reference genome. Nat Biotechnol 29(7): 644-652. doi: 10.1038/nbt.1883. PMID: 21572440. Guo, R., Mao, Y.R., Cai, J.R., Wang, J.Y., Wu, J., and Qiu, Y.X. 2014. Characterization and cross-species transferability of EST-SSR markers developed from the doi:10.1007/s11032-014-0134-z. Illumina platform. PLoS One 13(2): e0192610. doi.:10.1371/journal.pone.0192610. PMID: 29451882. 

SCoT markers. Biochem Syst Ecol 57: 338-344. doi:10.1016/j.bse.2014.09.016.

Kawata, J., Kameda, M., and Miyazawa, M. 2007. Constituents of essential oil from the dried fruits and stems of Akebia quinata (Thunb.) Decne. J Oleo Sci 56(2): 59-63. doi:10.5650/jos.56.59. PMID: 17898464. Japan. J NAT MED 63(3): 368-374. doi:10.1007/s11418-009-0318-8. PMID: 19277830. 28(4): 497-506. 
Li, L., Xu, Q., and Yao, X. 2019. Microsatellite analysis reveals the resilience of genetic diversity within extant populations of three Akebia species to chronic forest fragmentation in China. Plant Ecol 220(1): 69-81. doi:10.1007/s11258-018-00903-y.

Li, L., Yao, X.H., Chen X.Z., and Huang, H.W. 2009. Development and characterization of microsatellite loci in Chinese medicinal plant Akebia trifoliate ssp. australis and cross-species amplification in closely related taxa. Conserv Genet 10(4): 959-962. doi:10.1007/s10592-008-9666-2. during1960-2013. Prog Geog 34(3): 354-363. e0120615. doi:10.1371/journal.pone.0120615. PMID: 25799271.

Liu, G.Y., Ma, S.C., Zheng, J., Zhang, J., and Lin, R.C. 2007. Two New Triterpenoid Saponins from Akebia quinata (Thunb.) Decne. JIPB 49(2): $196-201$. doi:10.1093/bioinformatics/bti282. 
using microsatellite markers by restriction-associated DNA sequencing. Genes 9(4): 218-233. doi:10.3390/genes9040218. PMID: 29673217.

Luo, X., Cao, S., Hao, Z., Hou, L., Cao, D., Zhang, J., et al. 2018. Analysis of genetic structure in a large sample of pomegranate (Punica granatum L.) using fluorescent SSR markers. J Hortic Sci Biotech 93(6): 659-665. doi:10.1080/14620316.2018.1432994.

Ma, S., Dong, W., Lyu, T., and Lyu, Y. 2019. An RNA sequencing transcriptome analysis and development of EST-SSR markers in Chinese hawthorn through Illumina sequencing. Forests 10(2): 82-96. doi:10.3390/f10020082.

Mao, Y., Zhang, Y., Xu, C., and Qiu, Y. 2016. Comparative transcriptome resources of two Dysosma species (Berberidaceae) and molecular evolution of the CYP719A gene in Podophylloideae. Mol Ecol Resour 16(1): 228-241. doi:10.1111/1755-0998.12415.

Nguyen, N., Suokas, M., Karppinen, K., Vuosku, J., Jaakola, L., and Häggman, H. 2018. Recognition of candidate transcription factors related to bilberry fruit ripening by de novo transcriptome and qRT-PCR analyses. Sci Rep 8(1): 9943. doi:10.1038/s41598-018-28158-7. PMID: 29967355.

Niu, J., Cao, D., Li, H., Xue, H., Chen, L., Liu, B., et al. 2018. Quantitative proteomics of pomegranate varieties with contrasting seed hardness during seed development stages. Tree Genet Genome 14(1): 14-25. doi:10.1007/s11295-018-1229-1.

Ni, J. L., Zhu, A. G., Wang, X. F., Xu, Y., Sun, Z. M., Chen, J. H., et al. 2018. Genetic diversity and population structure of ramie (Boehmeria nivea L.). Ind Crop 
Prod 115: 340-347. doi:10.1016/j.indcrop.2018.01.038.

Ohbayashi, K., Ishikawa, N., Hodoki, Y., Okada, Y., and Shimada, M. 2019. Rapid development and characterization of EST-SSR markers for the honey locust seed beetle, Megabruchidius dorsalis (Coleoptera: Bruchidae), using de novo transcriptome analysis based on next-generation sequencing. Appl Entomol Zool 54(1): 141-145. doi:10.1007/s13355-019-00605-5.

Pritchard, J. 2000. Inference of population structure using multilocus genotype data. Genetics 155(2): 945-959. PMID: 10835412.

Shi, T., Li, R., Chen, Q., Li, Y., Pan, F., and Chen, Q. 2017. De novo sequencing of seed transcriptome and development of genic-SSR markers in common buckwheat (Fagopyrum esculentum). Mol Breeding 37(12): 147-161. doi:10.1007/s11032-017-0743-4.

Sun, Z.X., Ye, L.J., Zhang, F., Hu, W., Fan, D.M., and Zhang, Z.Y. 2016. Development of microsatellite markers for Sargentodoxa cuneata (Lardizabalaceae) using next-generation sequencing technology. Appl Plant Sci 4(5): 1-4. doi:10.3732/apps.1600003. PMID: 27213128.

Wang, J., Ren, H., Xu, Q.L., Zhou, Z.Y., Wu, P., Wei, X.Y., et al. 2015. Antibacterial oleanane-type triterpenoids from pericarps of Akebia trifoliata. Food Chem 168: 623-629. doi:10.1016/j.foodchem.2014.07.105. PMID: 25172756.

Wang, J., Xu, Q.L., Zheng, M.F., Ren, H., Lei, T., Wu, P., et al. 2014. Bioactive 30-noroleanane triterpenes from the pericarps of Akebia trifoliata. Molecules 19(4): 
4301-4312. Doi: 10.3390/molecules19044301

Wang, Z.Y., Fang, B.P., Chen, J.Y., Zhang, X.J., Luo, Z.X., Huang, L.F., et al. 2010. De novo assembly and characterization of root transcriptome using Illumina

311 paired-end sequencing and development of cSSR markers in sweetpotato (Ipomoea batatas). BMC Genom 11: 726-739. doi: 10.1186/1471-2164-11-726.

312 Wróblewska, A. 2014. Genetic diversity and spatial genetic structure of Chamaedaphne calyculata (Ericaceae) at the western periphery in relation to its main continuous range in Eurasia. Folia Geobot 49(2): 193-208. doi:10.1007/s12224-013-9165-1.

Wu, H., Chen, D., Li, J., Yu, B., Qiao, X.Y., Huang, H.L., et al. 2013. De novo characterization of leaf transcriptome using 454 sequencing and development of EST-SSR markers in tea (Camellia Sinensis). Plant Mol Biol Rep 31(3): 524-538. doi:10.1007/s11105-012-0519-2.

Xing, W., Liao, J., Cai, M., Xia, Q.F., Liu, Y., Zeng, W., et al. 2017. De novo assembly of transcriptome from Rhododendron latoucheae Franch. using Illumina sequencing and development of new EST-SSR markers for genetic diversity analysis in Rhododendron. Tree Genet Genomes 13(3): 53-66.

318 doi:10.1007/s11295-017-1135-y.

Xu, J., Li, A., Wang, X., and Qi, J. 2013. Genetic diversity and phylogenetic relationship of kenaf (Hibiscus cannabinus L.) accessions evaluated by SRAP and ISSR. 
Yang, H., Liu, H., Shi, X., Ge, F., Zhao, Z., and Luo, H. 2016. Transcriptome analysis of Akebia trifoliate in ripening process. J Mountain Agrie Biol 35(2): 46-51.

Yang, Z., Dai, Z., Xie, D., Chen, J., Tang, Q., Cheng, C., et al. 2018. Development of an indel polymorphism database for jute via comparative transcriptome analysis. Genome 61(5): 323-327. doi:10.1139/gen-2017-0191. PMID: 29420906.

Zhou,Y., Jie, B., Wu, G., Cao, Y., and Wu, W. 2017. Research progress of Akebia trifoliata (Thunb.) Koidz. Agr Biotech 6(6): 1-8.

Zou, S., Yao, X., Zhong, C., Zhao, T., and Huang, H. 2018. Genetic analysis of fruit traits and selection of superior clonal lines in Akebia trifoliate (Lardizabalaceae).

Euphytica 214(7): 111-119. doi:10.1007/s10681-018-2198-z. 
Tables

Table 1. Sampling details of $88 \mathrm{~A}$. trifoliate accessions from different regions used in the present study for SSR development and characterization.

334 Table 2. Summary of sequencing data of $A$. trifoliate transcriptome

Table 3. The statistical data of unigenes annotation

336 Table 4. Summary of simple sequence repeats (SSRs) identified from the A. trifoliate material

337 Table 5. Characteristics of 49 SSR primers that amplified successfully and exhibited polymorphism in $A$. trifoliate 


\section{Figure captions}

345 Fig. 1. Geographical distribution of collected A. trifoliate accessions

346 Fig. 2. The frequencies of SSR repeat motif, and the length of repeats in A. trifoliate. (A) The frequencies of the length of repeats. (B) The distribution of the SSR

repeat motif.

Fig. 3. Distribution of the number of repeats and principal type in SSR motif in A. trifoliate. (A) The distribution of the number of repeats. (B) The number of principal type in SSR motif

Fig. 4. Phylogenetic tree of 88 A. trifoliate accessions. Blue lines: Clade I; Green lines: Clade II; Purple lines: Clade III; Red lines: Clade IV

Fig. 5. Population structure analysis of 88 A. trifoliate accessions. (A) Population structure based on $\mathrm{K}=2$ and 4 . When $\mathrm{K}=4$, Red: Group 1; green: Group 2; blue: 
Supplementary Fig. 1 The size distribution of the assembled transcript and unigene sequences of $A$. trifoliate

357 Supplementary Fig. 2 Polymorphism validation of microsatellite primer P009 and P023 in 88 individuals of A. trifoliate Lane M 100bp DNA marker.

Supplementary Table S1 Main repeat motif in the different type of repeat

359 Supplementary Table S2 Characterization of all microsatellite loci, described by ID, forward and reverse primer sequences, Tm, size, product size (bp), start and end of product size. 
Table 1. Sampling details of 88 A. trifoliate accessions from different regions used in the present study for SSR development and characterization

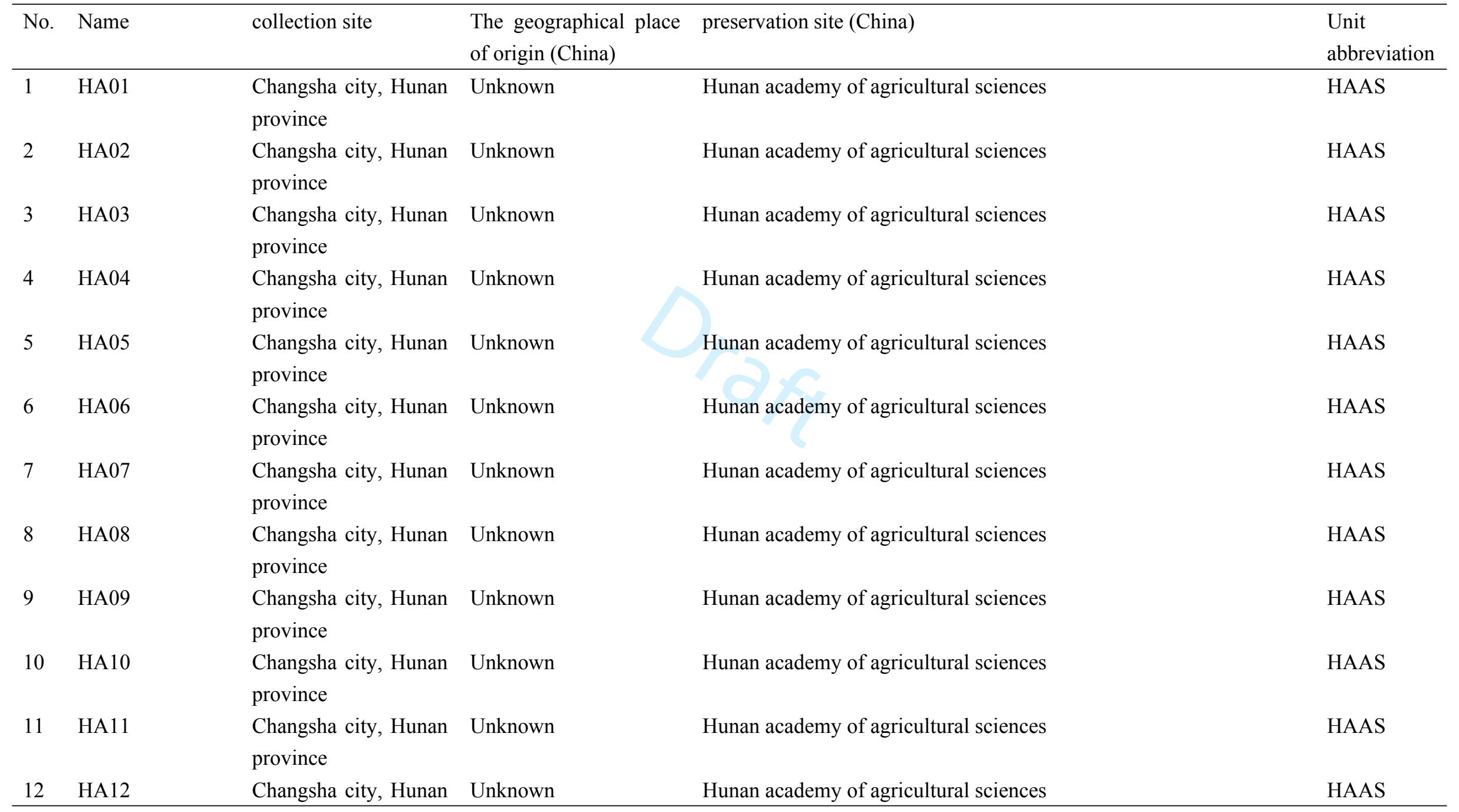




\section{province}

\begin{tabular}{|c|c|c|c|c|c|}
\hline & & & & & \\
\hline 13 & HA13 & $\begin{array}{l}\text { Changsha city, Hunan } \\
\text { province }\end{array}$ & Unknown & Hunan academy of agricultural sciences & HAAS \\
\hline 14 & HA14 & $\begin{array}{l}\text { Changsha city, Hunan } \\
\text { province }\end{array}$ & Unknown & Hunan academy of agricultural sciences & HAAS \\
\hline 15 & HA15 & $\begin{array}{l}\text { Changsha city, Hunan } \\
\text { province }\end{array}$ & Unknown & Hunan academy of agricultural sciences & HAAS \\
\hline 16 & HA16 & $\begin{array}{l}\text { Changsha city, Hunan } \\
\text { province }\end{array}$ & Unknown & Hunan academy of agricultural sciences & HAAS \\
\hline 17 & HA17 & $\begin{array}{l}\text { Changsha city, Hunan } \\
\text { province }\end{array}$ & Unknown & Hunan academy of agricultural sciences & HAAS \\
\hline 18 & HA18 & $\begin{array}{l}\text { Changsha city, Hunan } \\
\text { province }\end{array}$ & Unknown & Hunan academy of agricultural sciences & HAAS \\
\hline 19 & HA19 & $\begin{array}{l}\text { Changsha city, Hunan } \\
\text { province }\end{array}$ & Unknown & Hunan academy of agricultural sciences & HAAS \\
\hline 20 & JF01 & $\begin{array}{l}\text { Nanchang city, } \\
\text { Jiangxi province }\end{array}$ & Unknown & Jiangxi academy of forestry & JXAF \\
\hline 21 & JF02 & $\begin{array}{l}\text { Nanchang city, } \\
\text { Jiangxi province }\end{array}$ & Unknown & Jiangxi academy of forestry & JXAF \\
\hline 22 & ShangraoNo.1 & $\begin{array}{l}\text { Shangrao city, Jiangxi } \\
\text { province }\end{array}$ & $\begin{array}{l}\text { Shangrao city, } \\
\text { Jiangxi province }\end{array}$ & Jiangxi academy of forestry & JXAF \\
\hline 23 & JF04 & $\begin{array}{l}\text { Nanchang city, } \\
\text { Jiangxi province }\end{array}$ & Unknown & Jiangxi academy of forestry & JXAF \\
\hline 24 & JF05 & $\begin{array}{l}\text { Nanchang city, } \\
\text { Jiangxi province }\end{array}$ & Unknown & Jiangxi academy of forestry & JXAF \\
\hline 25 & JF06 & Nanchang & Unknown & Jiangxi academy of forestry & JXAF \\
\hline
\end{tabular}




\begin{tabular}{|c|c|c|c|c|c|}
\hline & & Jiangxi province & & & \\
\hline 26 & JF07 & $\begin{array}{l}\text { Nanchang city, } \\
\text { Jiangxi province }\end{array}$ & Unknown & Jiangxi academy of forestry & JXAF \\
\hline 27 & JF08 & $\begin{array}{l}\text { Nanchang city, } \\
\text { Jiangxi province }\end{array}$ & Unknown & Jiangxi academy of forestry & JXAF \\
\hline 28 & JF09 & $\begin{array}{l}\text { Nanchang city, } \\
\text { Jiangxi province }\end{array}$ & Unknown & Jiangxi academy of forestry & JXAF \\
\hline 29 & $\mathrm{JF} 10$ & $\begin{array}{l}\text { Nanchang city, } \\
\text { Jiangxi province }\end{array}$ & Unknown & Jiangxi academy of forestry & JXAF \\
\hline 30 & JF11 & $\begin{array}{l}\text { Nanchang city, } \\
\text { Jiangxi province }\end{array}$ & Unknown & Jiangxi academy of forestry & JXAF \\
\hline 31 & ShangraoNo. 2 & $\begin{array}{l}\text { Shangrao city, Jiangxi } \\
\text { province }\end{array}$ & $\begin{array}{l}\text { Shangrao city, Jiangxi } \\
\text { province }\end{array}$ & Jiangxi academy of forestry & JXAF \\
\hline 32 & NingguoNo.1 & $\begin{array}{l}\text { Nanchang city, } \\
\text { Jiangxi province }\end{array}$ & $\begin{array}{l}\text { Ningguo city, Anhui } \\
\text { province }\end{array}$ & Jiangxi academy of forestry & JXAF \\
\hline 35 & JF16 & $\begin{array}{l}\text { Nanchang city, } \\
\text { Jiangxi province }\end{array}$ & Unknown & Jiangxi academy of forestry & JXAF \\
\hline 36 & JF17 & $\begin{array}{l}\text { Nanchang city, } \\
\text { Jiangxi province }\end{array}$ & Unknown & Jiangxi academy of forestry & JXAF \\
\hline 37 & ChangdeNo.1 & $\begin{array}{l}\text { Changde city, Hunan } \\
\text { province }\end{array}$ & $\begin{array}{l}\text { Changde city, Hunan } \\
\text { province }\end{array}$ & Institute of bast fiber crops, Chinese academy of agricultural science & IBFC \\
\hline 38 & ChangdeNo.2 & Changde city, Hunan & Changde city, Hunan & Institute of bast fiber crops, Chinese academy of agricultural science & IBFC \\
\hline
\end{tabular}




\section{province province}

39 ChangdeNo.3 Changde city, Hunan Changde city, Hunan Institute of bast fiber crops, Chinese academy of agricultural science IBFC province province

40 ChangdeNo.4 Changde city, Hunan Changde city, Hunan Institute of bast fiber crops, Chinese academy of agricultural science IBFC province province

41 LuoyangNo.1 Luoyang city, Henan Luoyang city, Henan Institute of bast fiber crops, Chinese academy of agricultural science IBFC province province

42 LuoyangNo.2 Luoyang city, Henan Luoyang city, Henan Institute of bast fiber crops, Chinese academy of agricultural science IBFC province province

43 LuoyangNo.3 Luoyang city, Henan Luoyang city, Henan Institute of bast fiber crops, Chinese academy of agricultural science IBFC province province

44 LuoyangNo.4 Luoyang city, Henan Luoyang city, Henan Institute of bast fiber crops, Chinese academy of agricultural science IBFC province province

45 LuoyangNo.5 Luoyang city, Henan Luoyang province province

46 LuoyangNo.6 Luoyang city, Henan Luoyang city, Henan Institute of bast fiber crops, Chinese academy of agricultural science IBFC province province

47 LuoyangNo.7 Luoyang city, Henan Luoyang city, Henan Institute of bast fiber crops, Chinese academy of agricultural science IBFC

48 LuoyangNo.8 Luoyang city, Henan Luoyang city, Henan Institute of bast fiber crops, Chinese academy of agricultural science IBFC province province

49 LuoyangNo.9 Luoyang city, Henan Luoyang city, Henan Institute of bast fiber crops, Chinese academy of agricultural science IBFC province province

50 LuoyangNo.10 Luoyang city, Henan Luoyang city, Henan Institute of bast fiber crops, Chinese academy of agricultural science IBFC province province

51 Luoyang No.11 Luoyang city, Henan Luoyang city, Henan Institute of bast fiber crops, Chinese academy of agricultural science IBFC 


\begin{tabular}{|c|c|c|c|c|c|c|}
\hline & & province & province & & & \\
\hline 52 & DengfengNo.1 & $\begin{array}{l}\text { Dengfeng city, Henan } \\
\text { province }\end{array}$ & $\begin{array}{l}\text { Dengfeng city, } \\
\text { province }\end{array}$ & Henan & Institute of bast fiber crops, Chinese academy of agricultural science & $\mathrm{IBFC}$ \\
\hline 53 & DengfengNo.2 & $\begin{array}{l}\text { Dengfeng city, Henan } \\
\text { province }\end{array}$ & $\begin{array}{l}\text { Dengfeng city, } \\
\text { province }\end{array}$ & Henan & Institute of bast fiber crops, Chinese academy of agricultural science & $\mathrm{IBFC}$ \\
\hline 54 & DengfengNo.3 & $\begin{array}{l}\text { Dengfeng city, Henan } \\
\text { province }\end{array}$ & $\begin{array}{l}\text { Dengfeng city, } \\
\text { province }\end{array}$ & Henan & Institute of bast fiber crops, Chinese academy of agricultural science & IBFC \\
\hline 55 & DengfengNo.4 & $\begin{array}{l}\text { Dengfeng city, Henan } \\
\text { province }\end{array}$ & $\begin{array}{l}\text { Dengfeng city, } \\
\text { province }\end{array}$ & Henan & Institute of bast fiber crops, Chinese academy of agricultural science & IBFC \\
\hline 56 & DengfengNo.5 & $\begin{array}{l}\text { Dengfeng city, Henan } \\
\text { province }\end{array}$ & $\begin{array}{l}\text { Dengfeng city, } \\
\text { province }\end{array}$ & Henan & Institute of bast fiber crops, Chinese academy of agricultural science & IBFC \\
\hline 57 & DengfengNo.6 & $\begin{array}{l}\text { Dengfeng city, Henan } \\
\text { province }\end{array}$ & $\begin{array}{l}\text { Dengfeng city, } \\
\text { province }\end{array}$ & Henan & Institute of bast fiber crops, Chinese academy of agricultural science & $\mathrm{IBFC}$ \\
\hline 58 & DengfengNo.7 & $\begin{array}{l}\text { Dengfeng city, Henan } \\
\text { province }\end{array}$ & $\begin{array}{l}\text { Dengfeng city, } \\
\text { province }\end{array}$ & Henan & Institute of bast fiber crops, Chinese academy of agricultural science & $\mathrm{IBFC}$ \\
\hline 61 & DengfengNo.10 & $\begin{array}{l}\text { Dengfeng city, Henan } \\
\text { province }\end{array}$ & $\begin{array}{l}\text { Dengfeng city, } \\
\text { province }\end{array}$ & Henan & Institute of bast fiber crops, Chinese academy of agricultural science & IBFC \\
\hline 62 & DengfengNo.11 & $\begin{array}{l}\text { Dengfeng city, Henan } \\
\text { province }\end{array}$ & $\begin{array}{l}\text { Dengfeng city, } \\
\text { province }\end{array}$ & Henan & Institute of bast fiber crops, Chinese academy of agricultural science & IBFC \\
\hline 63 & DengfengNo.12 & $\begin{array}{l}\text { Dengfeng city, Henan } \\
\text { province }\end{array}$ & $\begin{array}{l}\text { Dengfeng city, } \\
\text { province }\end{array}$ & Henan & Institute of bast fiber crops, Chinese academy of agricultural science & IBFC \\
\hline 64 & DengfengNo.13 & Dengfeng city, Henan & Dengfeng city, & Henan & Institute of bast fiber crops, Chinese academy of agricultural science & IBFC \\
\hline
\end{tabular}




\section{province province}

65 DengfengNo.14 Dengfeng city, Henan Dengfeng city, Henan Institute of bast fiber crops, Chinese academy of agricultural science IBFC province province

66 DazhouNo.1 Dazhou city, Sichuan Dazhou city, Sichuan Institute of bast fiber crops, Chinese academy of agricultural science IBFC province province

67 DazhouNo.2 Dazhou city, Sichuan Dazhou city, Sichuan Institute of bast fiber crops, Chinese academy of agricultural science province province

68 DazhouNo.3 Dazhou city, Sichuan Dazhou city, Sichuan Institute of bast fiber crops, Chinese academy of agricultural science province province

69 DazhouNo.4 Dazhou city, Sichuan Dazhou city, Sichuan Institute of bast fiber crops, Chinese academy of agricultural science province province

70 DazhouNo.5 Dazhou city, Sichuan Dazhou city, Sichuan Institute of bast fiber crops, Chinese academy of agricultural science province province

71 DazhouNo.6 Dazhou city, Sichuan Dazhou city, Sichuan Institute of bast fiber crops, Chinese academy of agricultural science province province

72 ZhangjiajieNo.1 Zhangjiajie city, Zhangjiajie city, Hunan Institute of bast fiber crops, Chinese academy of agricultural science Hunan province province

73 ZhangjiajieNo.2 Zhangjiajie city, Zhangjiajie city, Hunan Institute of bast fiber crops, Chinese academy of agricultural science Hunan province province

74 SanmingNo.1 Sanming city, Fujian Sanming city, Fujian Institute of bast fiber crops, Chinese academy of agricultural science province

75 EnshiNo.1 Enshi autonomous Enshi autonomous Institute of bast fiber crops, Chinese academy of agricultural science prefecture, Hubei prefecture, Hubei province province

76 EnshiNo.2 Enshi autonomous Enshi autonomous Institute of bast fiber crops, Chinese academy of agricultural science 


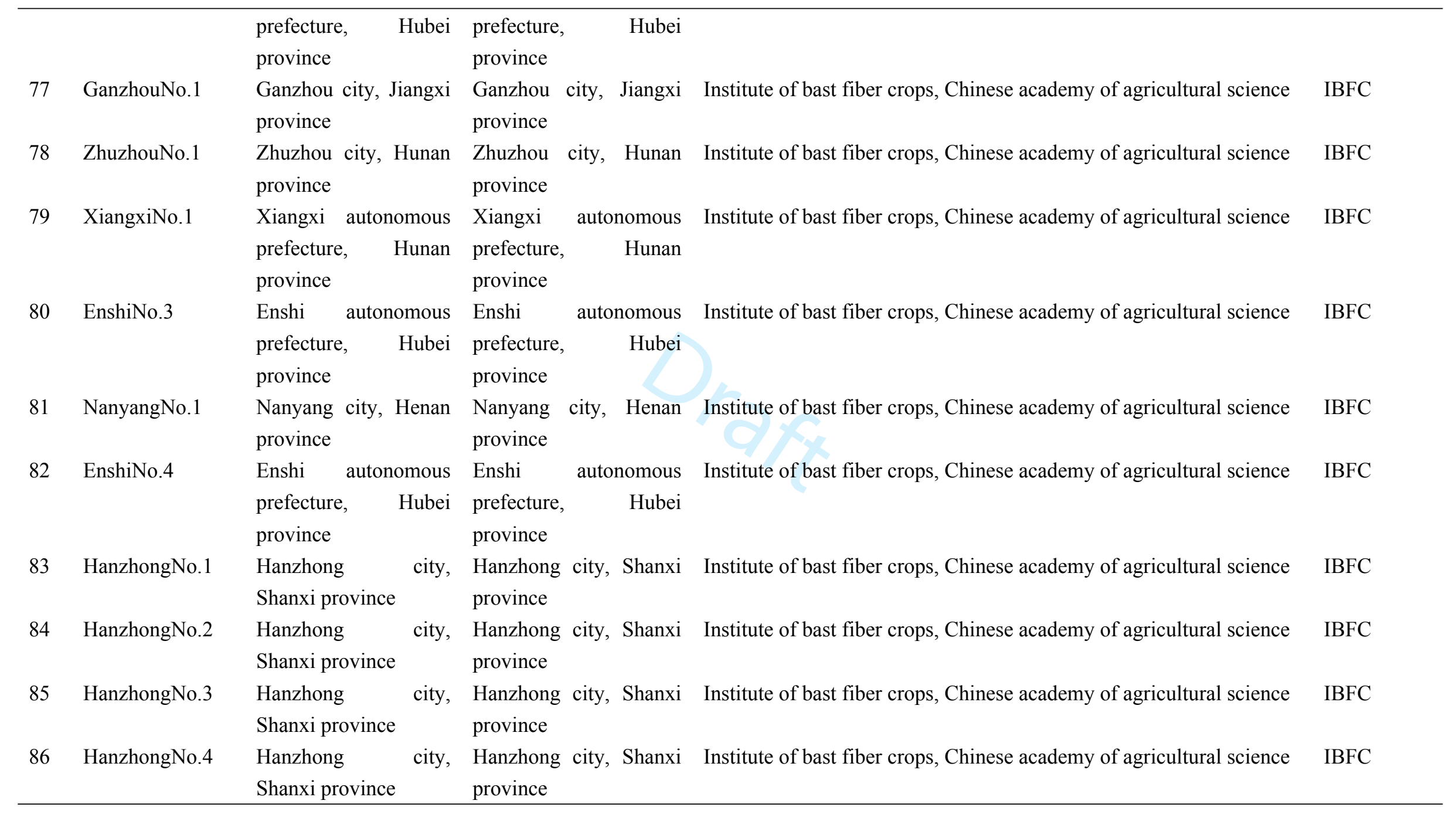


87 HanzhongNo.5

Hanzhong

city, Hanzhong city, Shanxi

Institute of bast fiber crops, Chinese academy of agricultural science

IBFC

Shanxi province province

88 EnshiNo.5

Enshi

autonomous Enshi

autonomous Institute of bast fiber crops, Chinese academy of agricultural science

IBFC prefecture

Hubei

prefecture, Hubei

province province 
Table 2. Summary of sequencing data of A. trifoliate transcriptome

\begin{tabular}{lc}
\hline Summary of the transcriptome & Number/length \\
\hline Raw reads & 50364426 \\
Total bases (Gb) & 7.555 \\
Clean reads & 25049450 \\
Clean bases (Gb) & 7.205 \\
Average size of clean reads (bp) & 143.81 \\
GC content (\%) & $45.10 \%$ \\
Q30 percentage (\%) & $92.56 \%$ \\
Transcripts & 101417 \\
N50 length (bp) & 1692 \\
Mean length (bp) & 983.28 \\
Unigenes & 63757 \\
N50 length (bp) & 1487 \\
Mean length (bp) & 769.89 \\
\hline
\end{tabular}


Table 3. The statistical data of unigenes annotation

\begin{tabular}{lcc}
\hline Annotated databases & $\begin{array}{l}\text { Number and ratio of annotated } \\
\text { unigenes }\end{array}$ & $\begin{array}{l}\text { The species provided most of } \\
\text { the annotation }\end{array}$ \\
\hline Pfam & 19661 & \\
Tremble & $(30.84 \%)$ & Arabidopsis thaliana \\
& 27772 & $(14552,63.8 \%)$ \\
eggNOG & $(43.56 \%)$ & \\
Swissport & 20917 & Nelumbo nucifera \\
GO & $(32.81 \%)$ & $(13247,47.70 \%)$ \\
KEGG & 19096 & \\
All & $(29.95 \%)$ & \\
Annotation ratio & 22798 & \\
\hline
\end{tabular}


Table 4. Summary of simple sequence repeats (SSRs) identified from the A. trifoliate material

\begin{tabular}{ll}
\hline SSR information & Number \\
\hline Total Number of sequences examined & 63757 \\
Total number of identified SSRs & 20169 \\
Number of sequences containing SSRs & 14519 \\
Number of sequences containing more than 1 SSR & 4036 \\
Number of SSRs with high quality & 9494 \\
Mono-nucleotide repeats & 2340 \\
Di-nucleotide repeats & 4021 \\
Tri-nucleotide repeats & 2850 \\
Tetra-nucleotide repeats & 207 \\
Penta-nucleotide repeats & 43 \\
Hexa-nucleotide repeats & 33 \\
\hline
\end{tabular}


Table 5. Characteristics of 49 SSR primers that amplified successfully and exhibited polymorphism in A. trifoliate

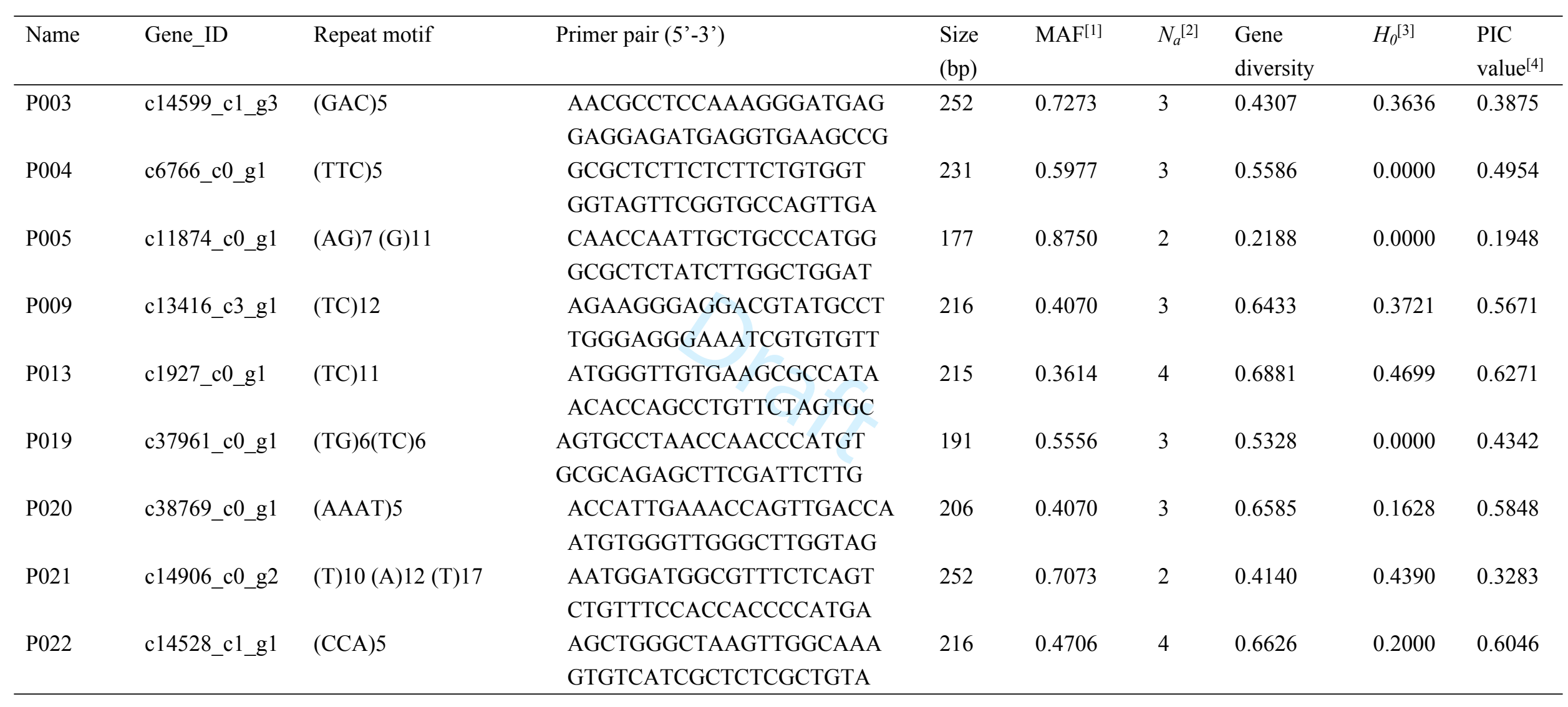

[1] Major allele frequency

${ }^{[2]}$ Number of alleles

[3] Observed heterozygosity

${ }^{[4]}$ Value of polymorphism information content 







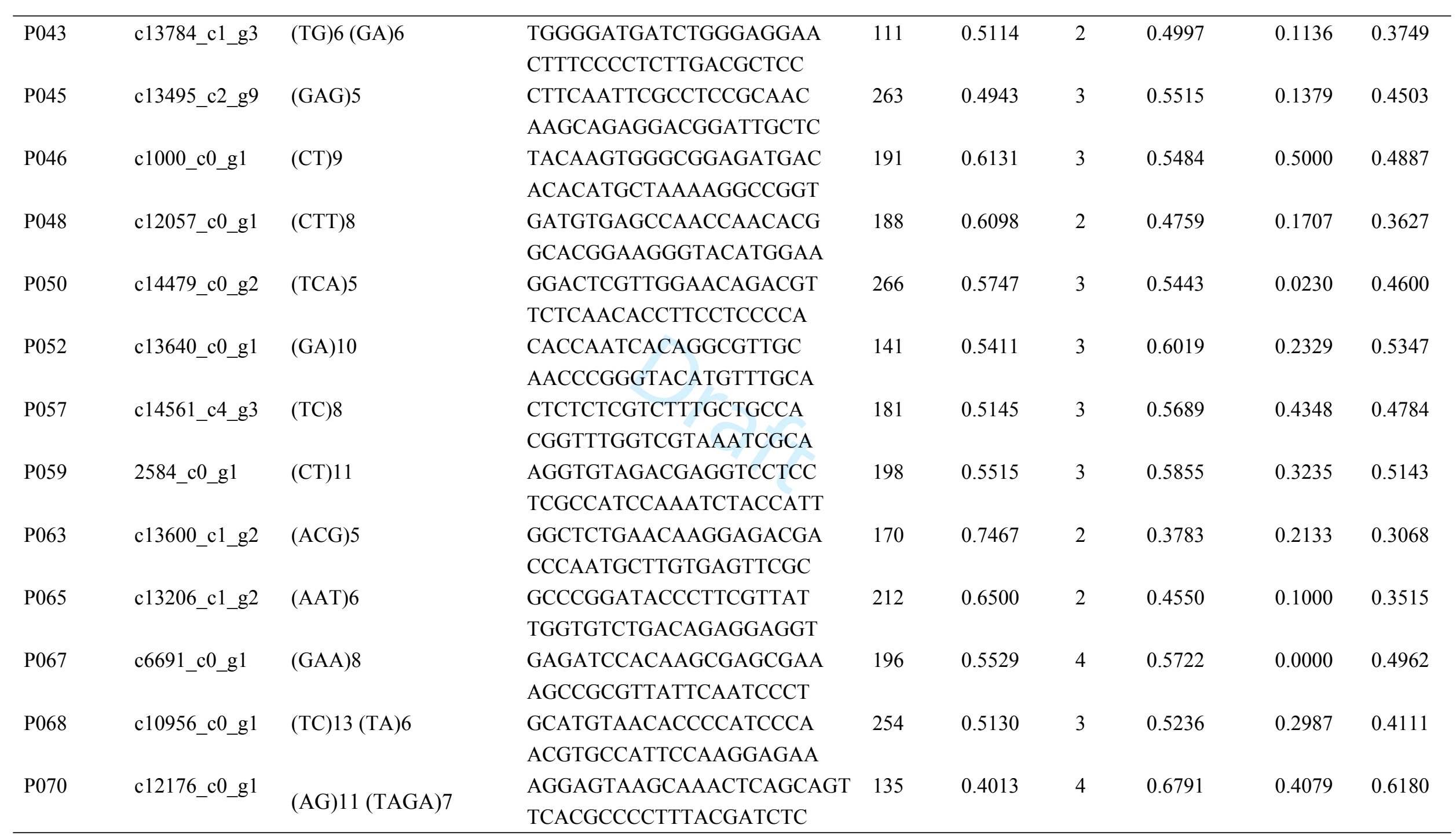




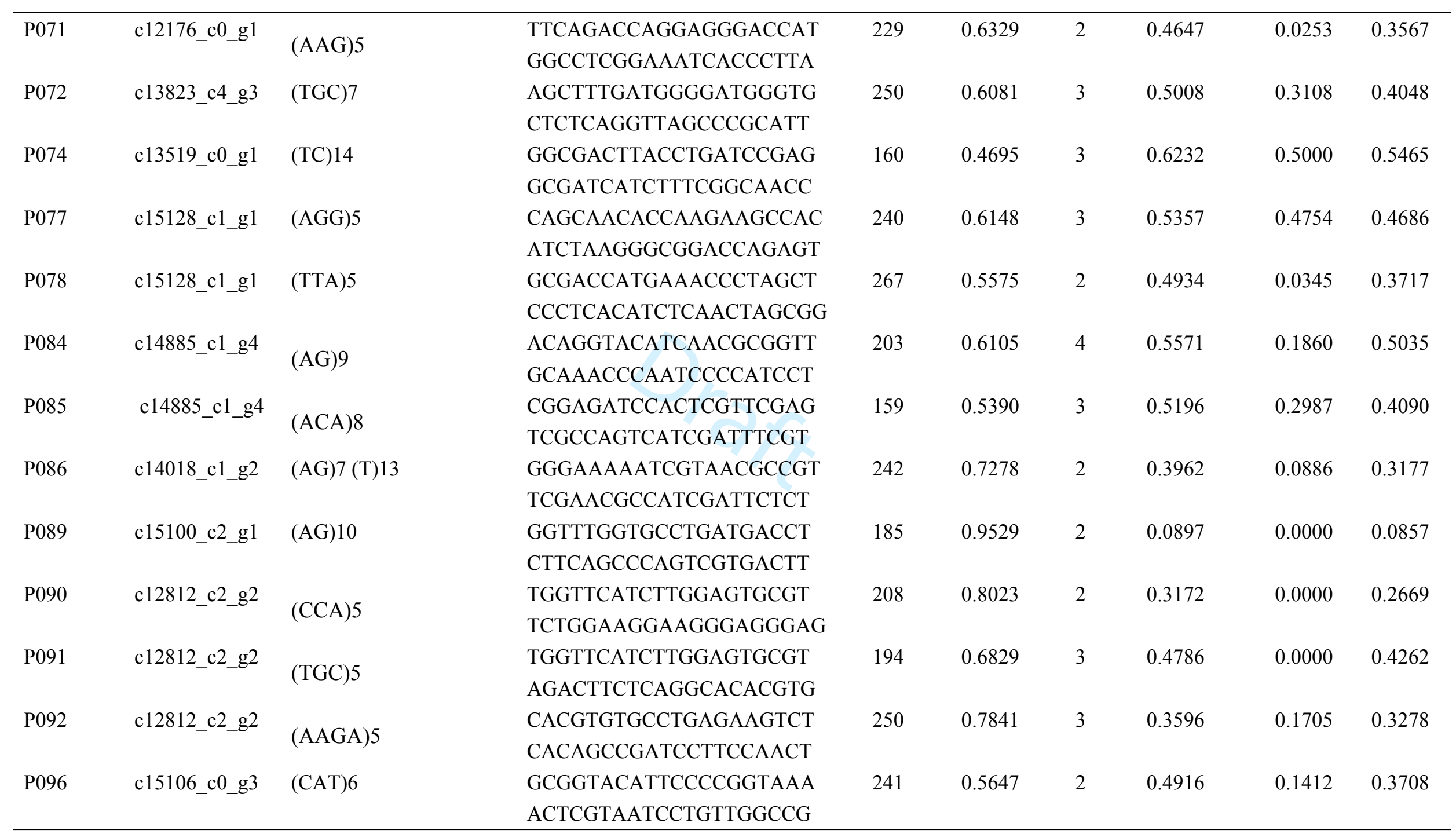




\begin{tabular}{|c|c|c|c|c|c|c|c|c|c|}
\hline P100 & c13025_c2_g1 & $(\mathrm{CAA}) 6$ & $\begin{array}{l}\text { GTTCGCGTGGGAAGAACAAC } \\
\text { ACCTTCTGATGCGCTTCGAA }\end{array}$ & 217 & 0.4651 & 4 & 0.6170 & 0.4070 & 0.5416 \\
\hline Mean & & & & & 0.6033 & 3 & 0.5020 & 0.2210 & 0.4261 \\
\hline
\end{tabular}




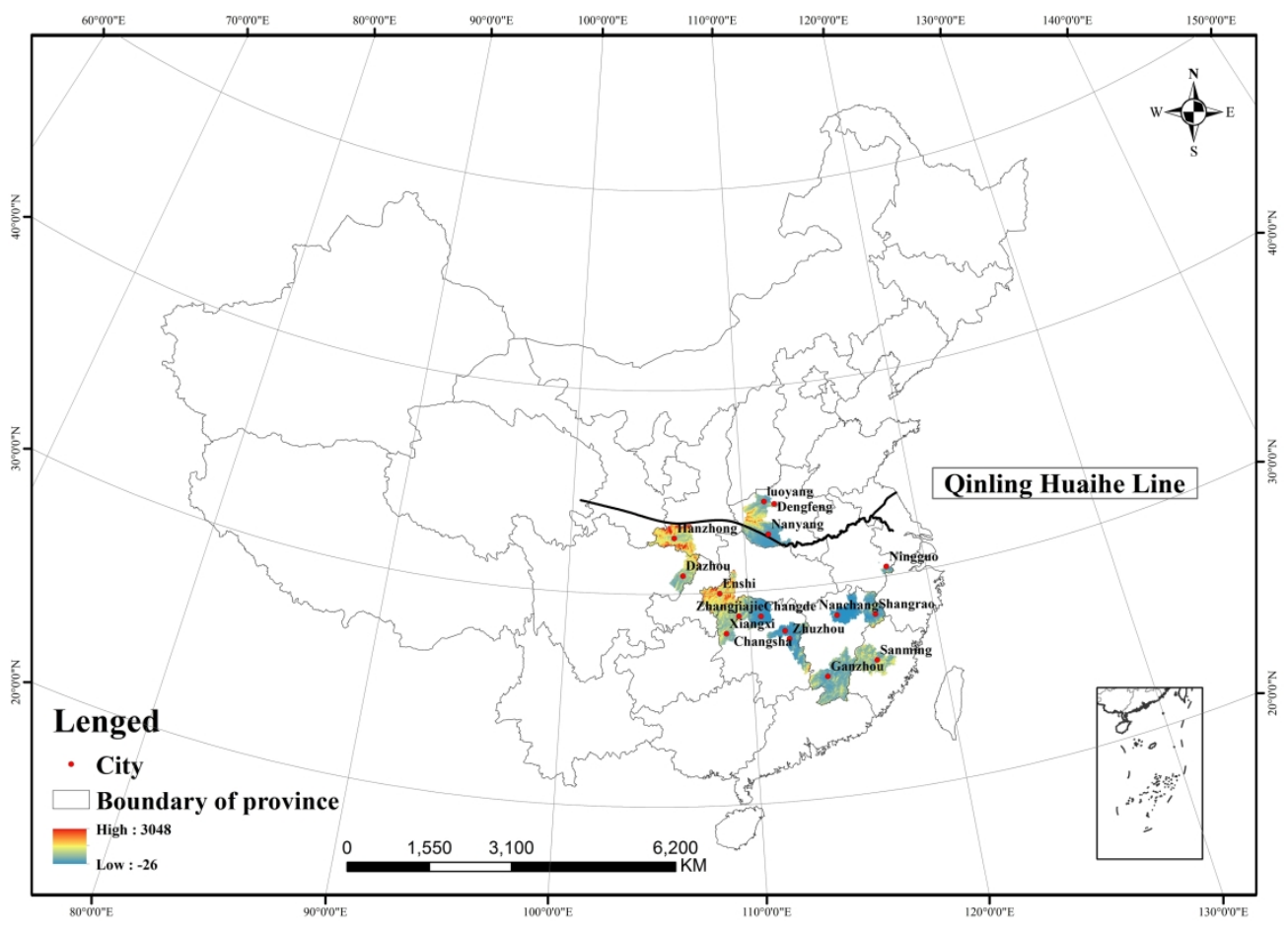

$297 \times 210 \mathrm{~mm}(300 \times 300$ DPI $)$ 

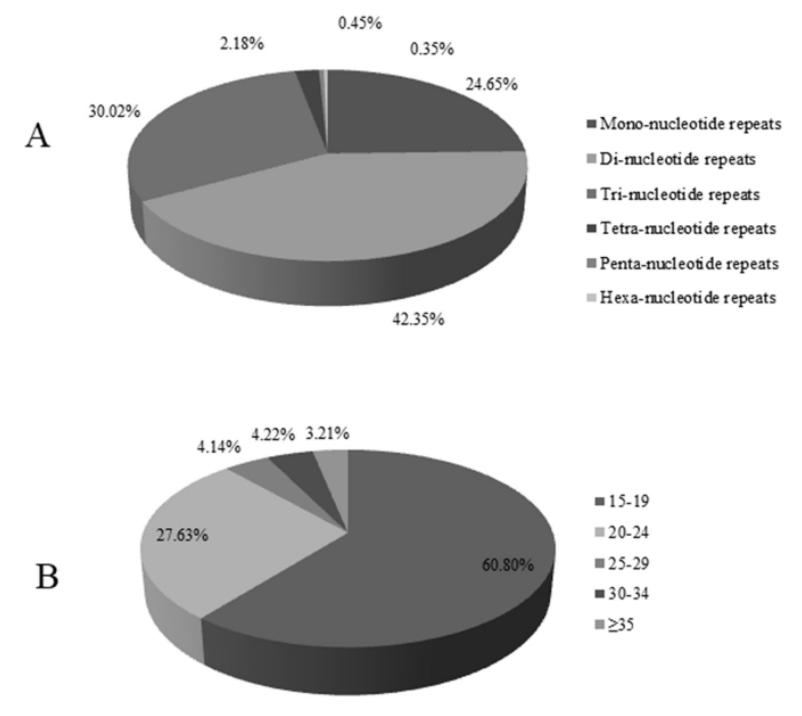

$254 \times 190 \mathrm{~mm}(300 \times 300 \mathrm{DPI})$

https://mc06.manuscriptcentral.com/genome-pubs 

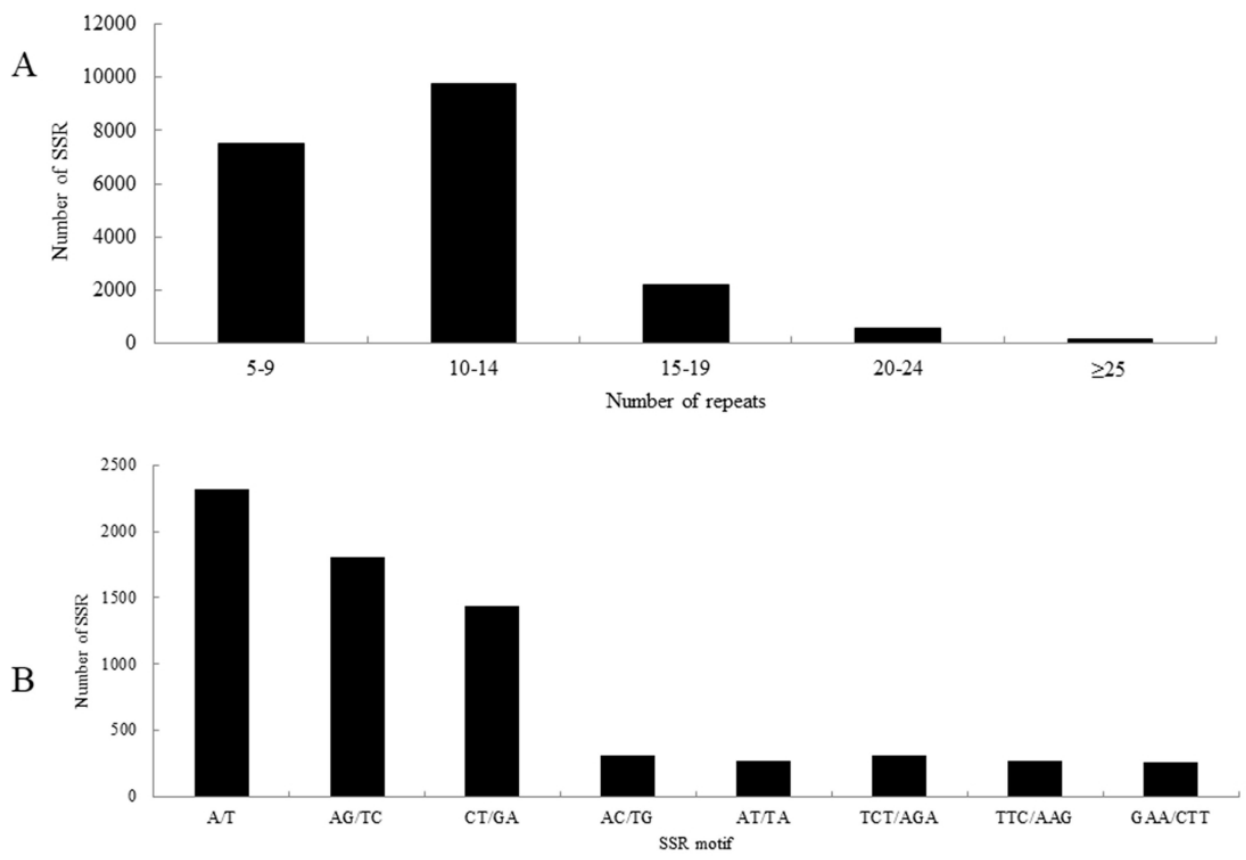

$254 \times 190 \mathrm{~mm}(300 \times 300$ DPI $)$ 


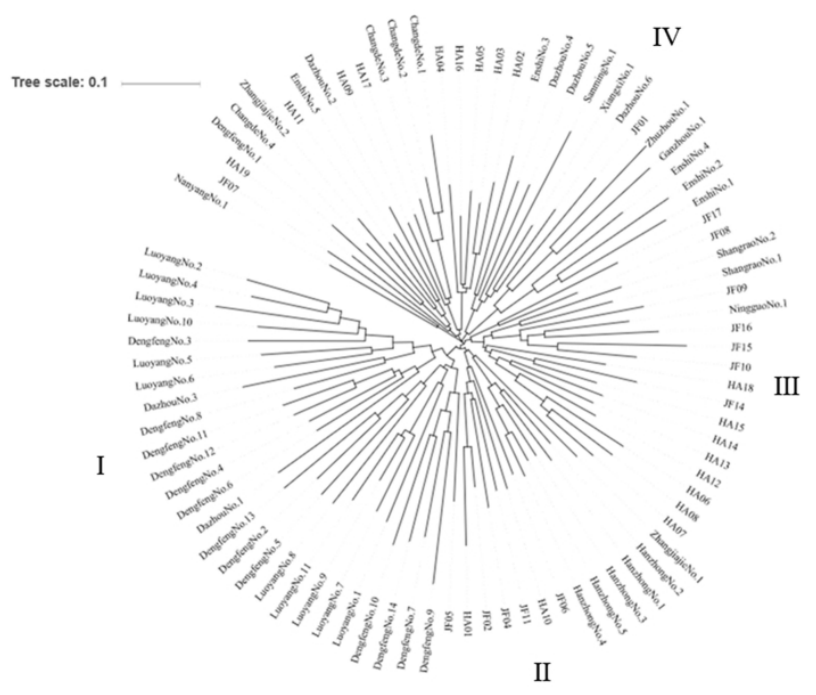

$338 \times 190 \mathrm{~mm}(300 \times 300 \mathrm{DPI})$ 


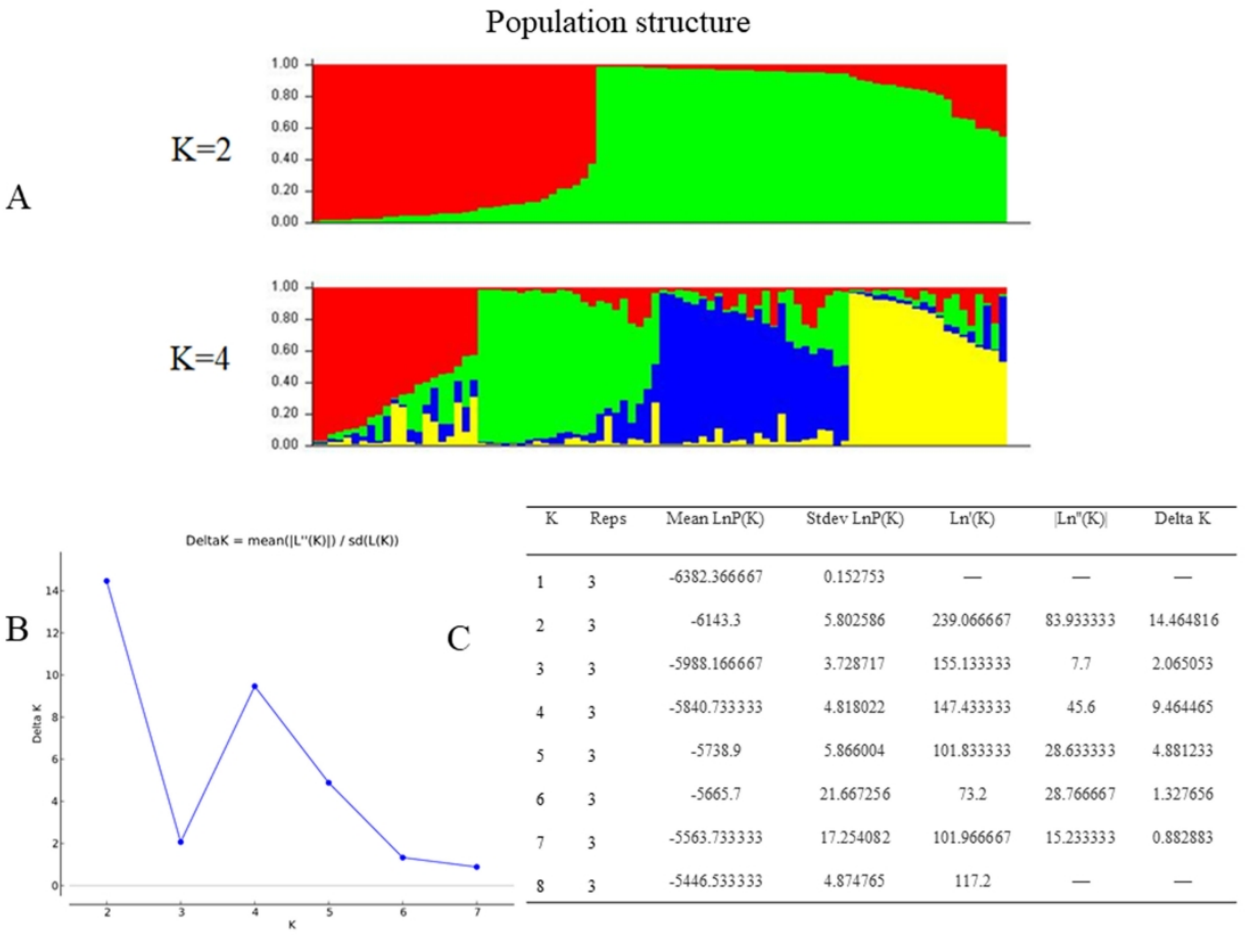

$254 \times 190 \mathrm{~mm}(300 \times 300$ DPI $)$ 